\title{
A FRICTIONLESS CONTACT ALGORITHM FOR DEFORMABLE BODIES*
}

\author{
OLIVIER PANTZ ${ }^{1}$
}

\begin{abstract}
This article is devoted to the presentation of a new contact algorithm for bodies undergoing finite deformations. We only address the kinematic aspect of the contact problem, that is the numerical treatment of the non-intersection constraint. In consequence, mechanical aspects like friction, adhesion or wear are not investigated and we restrict our analysis to the simplest frictionless case. On the other hand, our method allows us to treat contacts and self-contacts, thin or non-thin structures in a single setting.
\end{abstract}

Mathematics Subject Classification. 74B20, 74M15.

Received October 10, 2007. Revised January 13, 2009 and October 15, 2009.

Published online August 2, 2010.

\section{INTRODUCTION}

Industrial needs have stimulated the numerical simulation of mechanical contacts which has drawn a lot of attention over the last years. Many algorithms have been proposed in order to solve problems of growing complexity: contacts between rigid or (visco-)elastic bodies, submitted to small or finite deformations, with or without friction, adhesion or wear. In this article, we restrict ourself to the study of frictionless contact between deformable bodies undergoing large deformations. Such a goal may sound limited, but as it constitutes the elementary component of any other contact algorithm, it seems justified to study it for itself. If friction, wear or adhesion are mechanical properties that depend on the nature of the surfaces in contact, this is not the case for the kinematic condition of non-intersection.

The most common approach is known as the master/slave formulation. Let us briefly recall what it consists in. Consider two deformable bodies whose reference configurations are two open subsets of $\mathbb{R}^{n}(n=2$ or 3$), M_{1}$ and $M_{2}$. Let $\varphi_{i}: M_{i} \rightarrow \mathbb{R}^{n}(i=1,2)$ be their deformations. One of the two solids, say $M_{1}$, is called the master body, whereas the other one, i.e. $M_{2}$, is called the slave. We introduce the gap function $g_{\varphi}$, which maps every element $x$ of $M_{2}$ to the signed distance between $\varphi_{2}(x)$ and $\varphi_{1}\left(\partial M_{1}\right)$, defined by

$$
g_{\varphi}(x)=\left\{\begin{aligned}
\operatorname{dist}\left(\varphi_{2}(x), \varphi_{1}\left(\partial M_{1}\right)\right) & \text { if } \varphi_{2}(x) \notin \varphi_{1}\left(M_{1}\right) \\
-\operatorname{dist}\left(\varphi_{2}(x), \varphi_{1}\left(\partial M_{1}\right)\right) & \text { if } \varphi_{2}(x) \in \varphi_{1}\left(M_{1}\right) .
\end{aligned}\right.
$$

\footnotetext{
Keywords and phrases. Contact, frictionless, self-contact, elasticity, finite deformations.

* "Mathematical modelling and numerical simulation", F-EADS - École Polytechnique - INRIA.

1 CMAP, École Polytechnique, 91128 Palaiseau Cedex, France. olivier.pantz@polytechnique.org
} 
In order to prevent any overlapping between the two deformed solids, a constraint is applied to any element of the boundary of the slave body $M_{2}$. More precisely, it consists to impose that the signed distance between any element of $\varphi\left(\partial M_{2}\right)$ and $\varphi\left(\partial M_{1}\right)$ is non negative:

$$
g_{\varphi}(x) \geq 0 \text { for all } x \in \partial M_{2}
$$

A breakdown of this formulation can be found in Laursen's thesis [18,19] (see also [20]). This approach is confronted with two important difficulties. Firstly, it can not be directly applied neither to the case of selfcontacts (where $M_{1}=M_{2}$ and $\left.\varphi_{1}=\varphi_{2}\right)$ nor to thin structures $\left(\varphi_{i}: M_{i} \rightarrow \mathbb{R}^{n}\right.$ and $M_{i}$ is a submanifold of dimension $m<n)$. For instance, in the self-contact case, the constraint (1.1) is empty: the signed distance between an element of $\varphi_{1}\left(\partial M_{1}\right)$ and $\varphi_{1}\left(\partial M_{1}\right)$ is always zero. Moreover, even if we consider contact between two distinct bodies such that $\operatorname{det}\left(\nabla \varphi_{i}\right)>0$ almost everywhere, condition (1.1) is not sufficient to ensure nonintersection (see [25]). Secondly, the gap function is not everywhere differentiable with respect to $\varphi_{1}$ and $\varphi_{2}$. This lack of differentiability seems to be one the cause of the well known chatter phenomena, a loss of convergence in the numerical schemes (the computed solutions chatter around the equilibrium state). Several strategies have been developed in order to overcome those reefs. Puso and Laursen [28] notably proposed a regularization of the gap function, while mortar methods, used to integrate contact forces, also enable to improve convergence (see for instance [36]). Heinstein et al. [12] have adapted the master/slave approach to the study of thin structures and self-contacts. Their algorithm seems to be efficient in most common cases, but there is no guarantee it could correctly treat every situation. For a detailed presentation of the different strategies developed in this context, we refer to the Hallquist et al. article [11], which contains many references, and to the Kikuchi and Oden [16] or Wriggers [35] monographs (see also [17,34]). A different approach, called "material depth", has been proposed by Hirota et al. [13] to treat self-contacts, but it seems difficult to adapt to the case of thin structures. Other methods have been designed to treat the particular case of rigid bodies. The Non Smooth Contact Dynamic (NSCD) formulation has been introduced by Jean [14,15] and Moreau [24] for the study of granular media. For computing graphics purposes (see also Baraff [3,4], Baraff and Witkin [5,6]), Milenkovic and Schmidl [21] proposed an "Optimization Based Animation" (OBA) approach. However similar the NSCD and OBA methods are, they seem to have been developed independently.

If the master/slave formulation consists in a natural extension of the contact treatment between a deformable body and a rigid foundation, it has no complete theoretical justification. The main theoretical issues linked to the study of frictionless contacts deal with the definition of the set of admissible deformations, the existence of minimizers of the energy over this set and the derivation of the Euler-Lagrange equations. Ball [2] considered the case of hyperelastic bodies fixed on all of their boundaries, and whose stored energy grows to infinity as the determinant of the gradient of the deformation goes to zero. Assuming the polyconvexity of the stored energy function, he states an existence result to the minimization problem of the energy and proves that minimizers of the energy are injective almost everywhere: local injectivity implies global injectivity. Ciarlet and Nečas [7] extended his works to mixed boundary conditions (see also Tang [33], Giaquinta et al. [8,9]). Nevertheless their analysis does not apply to the study of thin structures. Gonzalez et al. [10] and Schuricht and von der Mosel [32] introduced the notion of global curvature in order to consider contacts and self-contacts between unidimensional structures (see also [29-31]). In a recent work [25], we have proposed a new modeling of the contacts and selfcontacts between deformable bodies, which seems physically relevant in the static case when $\operatorname{dim}\left(M_{i}\right)=m$ and $2 m \geq n$ (where $n$ is the dimension of the space where $M_{i}$ is injected, that is $\varphi_{i}$ is a mapping from $M_{i}$ into $\mathbb{R}^{n}$ ). This modeling relies on the introduction of a new set of admissible deformations. A topological constraint is introduced to ensure that no admissible deformation exhibits transversal (self)intersections.

In this article, we only address the numerical aspect of the problem, excluding any theoretical considerations. As we previously underline, the master/slave approach is not completely consistent when self-intersections or thin structures are involved. Even if it could be adapted in order to correctly manage such cases in most situations, this is done only to a costly development price: Many subtle tricks have to be introduced to this end. Instead of trying to adapt the master/slave approach, we adopt a totally different point of view, which allows us to consider contacts and self-contacts as well as contact between thin structures in a single setting. 


\section{Setting of the Problem}

Let us consider a family of connected deformable bodies $M=\left(M_{i}\right)$ moving in $\mathbb{R}^{n}(n=2$ or 3 ), such that for each index $i$, we have $n \geq \operatorname{dim}\left(M_{i}\right) \geq 1$. We denote by $\varphi_{i}: M_{i} \rightarrow \mathbb{R}^{n}$ the deformation of the body $M_{i}$, which maps each point $x$ of $M_{i}$ onto its position $\varphi_{i}(x)$ in $\mathbb{R}^{n}$. In the static case, the state of the system is completely described by the mapping $\varphi$ from $\cup_{i} M_{i}$ into $\mathbb{R}^{n}$ whose restriction to each body $M_{i}$ is its deformation $\varphi_{i}$.

We assume that we can associate to each state of the system an energy, and denote by $J$ the functional that maps every deformation $\varphi$ onto its energy. Our goal is to determine the minimum energy state, that is to find $\varphi \in \mathcal{A}(M)$ such that

$$
J(\varphi):=\inf _{\psi \in \mathcal{A}(M)} J(\psi)
$$

where $\mathcal{A}(M)$ is the set of deformations without self-intersection. It remains to give a mathematical definition to this set.

Remark 2.1. The family of bodies $M$ could not contain single points as we assume for each index $i$ the dimension of $M_{i}$ to be positive. The results we present in the following could be extended in order to take into account this particular class of bodies. As it is difficult to consider single material points as "deformable" bodies, we have chosen to exclude them from our analysis. It allows us to slightly simplify the presentation.

The master/slave approach consists in imposing constraint (1.1) to any couple of bodies of the collection $M$. Unfortunately, this constraint is empty if $M$ is made of a single body. Moreover, it can not be applied whenever $M$ contains more than one thin structure (it is still reasonable for one thin structure, chosen as the slave, and one solid of dimension equal to $n$, chosen as the master). In order to take these cases into account, we have to look for another definition. Firstly, let us recall that an embedding of $M$ into $\mathbb{R}^{n}$ is a regular one-to-one mapping from $M$ with values in $\mathbb{R}^{n}$, whose gradient $D \varphi$ is everywhere of maximal rank (that is equal to $m$ ). We denote by $\operatorname{Emb}\left(M ; \mathbb{R}^{n}\right)$ the set of embeddings of $M$ into $\mathbb{R}^{n}$, and define the admissible set $\mathcal{A}(M)$ as the closure of $\operatorname{Emb}\left(M ; \mathbb{R}^{n}\right.$ ) (for a topology depending on the energy $J$ ):

$$
\mathcal{A}(M)=\overline{\operatorname{Emb}\left(M ; \mathbb{R}^{n}\right)}
$$

We proved several properties of this set in [25,27]. In particular, it does not contain any deformation with transversal self-intersections. This definition enables to treat in a single setting contacts, self-contacts, thin or non-thin structures, however, it is implicit. Contrarily to the definition arising from the master/slave approach, it is not obvious to find out whether or not a given deformation belongs to the admissible set. In [25,27], we prove that any deformation of $\mathcal{A}(M)$ satisfies an explicit criterion. Moreover, we conjecture that this criterion is only fulfilled (in dimension $n=2$ ) by the elements of $\mathcal{A}(M)$. This allows to solve problem (2.1) by a penalization method (see [26]). Nevertheless, to our knowledge, there is currently no explicit definition of $\mathcal{A}(M)$ in the general case.

\section{An optimization Algorithm Under nONCONVEX CONSTRAints}

A major difficulty in the resolution of problem (2.1) is due to the strong nonlinearity introduced by the nonconvex constraint of non-interpenetration. There is no general method to directly solve such problems. On the other hand, efficient algorithms are available to minimize convex functions under convex constraints. Thus, a classical method for tackling this sort of problem is to recast it as a sequence of convex problems. With this in mind, two radically different options are conceivable. One consists in removing any constraint: We minimize on the set of all deformations and penalize the non-admissible ones. We have developed this strategy in [26]. Another approach consists in minimizing on a convex subsets of the admissible set. A local minimizer could thus be achieved by a recursive procedure. At each step, the energy is minimized over a convex "neighborhood" of the previous solution, included in the admissible set, until a fixed point is reached. This option has two advantages. Firstly, it requires neither the introduction of a penalization function nor the definition of an explicit characterization of the admissible set, which is to our knowledge an open problem in the general case. 
Secondly, no undesirable intersections have to be removed during the minimization process, which seems to be a difficult issue. To be more precise, our algorithm has three steps:

(1) Initialization of $\varphi_{0}$ by an admissible deformation;

(2) For all $n \geq 0$, we denote $\varphi_{n+1} \in T\left(\varphi_{n}\right)$, the solution of the minimization problem

$$
J\left(\varphi_{n+1}\right):=\inf _{\psi \in T\left(\varphi_{n}\right)} J(\psi)
$$

(3) $\operatorname{STOP}$ when $J\left(\varphi_{n+1}\right) \simeq J\left(\varphi_{n}\right)$,

where $T(\cdot)$ maps any admissible deformation $\psi$ to a convex subset $T(\psi)$ of the admissible deformations that contains the element $\psi$. Note that the stopping criterion is always reached as $J\left(\varphi_{n}\right)$ is a decreasing sequence, bounded from below (assuming the infimum is finite). If $\psi$ belongs to the interior of the admissible set, $T(\psi)$ will always be chosen as a (closed) neighborhood of $\psi$. On the contrary, if $\psi$ belongs to the boundary of the admissible set, it is not granted that any convex neighborhood of $\psi$ in $\mathcal{A}(M)$ does actually exist, since $\mathcal{A}(M)$ is not necessarily locally convex. Nevertheless, we will often refer to $T(\psi)$ as a "neighborhood" of $\psi$ by language abuse.

We will not apply this algorithm directly to the initial problem (2.1), but to a discretized version for which we have an explicit definition of the admissible set.

\section{Discretization}

We assume that the space of deformations can be discretized with $P_{1}$ Lagrangian elements or in other words that the set of all $P_{1}$ Lagrangian elements is dense in the space of deformation with finite energy. Let $\mathcal{T}_{h}$ be a regular mesh of $M$ - the parameter $h$ is the mesh size - and $X_{h}$ the space of $P_{1}$ Lagrange elements over this mesh,

$$
X_{h}=\left\{\psi \in \mathrm{C}^{0}\left(M ; \mathbb{R}^{n}\right):\left.\psi\right|_{T} \in P_{1}^{n}, \text { for all } T \in \mathcal{T}_{h}\right\},
$$

where $P_{1}$ is the set of polynomials of degree lower or equal to one. We introduce another parameter $\varepsilon$, the minimal distance we will impose between any disjoint elements of the mesh. Finally, the discretized set of admissible deformations is defined by

$$
\mathcal{A}_{h, \varepsilon}=\left\{\psi_{h} \in X_{h}: \operatorname{dist}\left(\psi_{h}\left(T_{1}\right), \psi_{h}\left(T_{2}\right)\right) \geq \varepsilon, \text { for all } T_{1} \text { and } T_{2} \in \mathcal{T}_{h} \text { such that } T_{1} \cap T_{2}=\emptyset\right\} .
$$

We set to solve the discretized minimization problem of $J$ over $\mathcal{A}_{h, \varepsilon}$

$$
\min _{v_{h} \in \mathcal{A}_{h, \varepsilon}} J\left(v_{h}\right)
$$

using the procedure introduced in the previous section. To this end, it remains to define the map $T$ that maps every element $\psi_{h}$ of $\mathcal{A}_{h, \varepsilon}$ onto a convex "neighborhood" $T\left(\psi_{h}\right)$ included in $\mathcal{A}_{h, \varepsilon}$. Problem (4.1) is an internal approximation of the initial problem (2.1) as

$$
\mathcal{A}=\overline{\bigcup_{h, \varepsilon} \mathcal{A}_{h, \varepsilon}}
$$

Remark 4.1. The definition of $\mathcal{A}_{h, \varepsilon}$ can be generalized by choosing a parameter $\varepsilon$ depending on the elements $T_{1}$ and $T_{2}$ of the mesh considered. Note that, in the definition of $\mathcal{A}_{h, \varepsilon}, T_{1}$ and $T_{2}$ can be any elements of the mesh $\mathcal{T}_{h}$, that is vertices, edges, triangles or tetrahedrons. 
It is not straightforward to extend our analysis to other space discretizations. Moreover, if the energy contains some bending terms, affine by parts maps do not have a finite energy. Nevertheless, different discretizations could be used for the contact constraint and for the energy and thus enable us to use our approach even if the set of all $P_{1}$ Lagrangian elements is not dense in the space of deformation with finite energy. To be more precise, let $Y_{h}$ be a discretized subset of the deformations of finite energy and $r_{h}$ be a linear interpolation operator from $Y_{h}$ into $X_{h}$, then the minimization problem

$$
\min _{r_{h}\left(v_{h}\right) \in \mathcal{A}_{h, \varepsilon}, v_{h} \in Y_{h}} J\left(v_{h}\right)
$$

can be solved using our algorithm and the map $T(\cdot)$ defined thereafter. However, the precision of the scheme will be limited by the contact discretization, which could be coarse in comparison with the one of the energy.

\subsection{Definition of a convex "neighborhood"}

In this section, we define the map $T(\cdot)$ that maps every admissible deformation $\psi_{h} \in \mathcal{A}_{h, \varepsilon}$ to a closed convex subset of $\mathcal{A}_{h, \varepsilon}$ containing $\psi_{h}$. Let us underline that there is a margin of freedom in the definition of $T$. Our choice is mostly guided by a wish for simplicity, others are conceivable. In order to avoid an unnecessarily complex formalism, we distinguish the two-dimensional case from the three-dimensional one.

\subsubsection{The two-dimensional case}

In the two-dimensional case, that is $n=2$, we define the map $T(\cdot)$ by

$$
\begin{aligned}
T\left(\psi_{h}\right)=\left\{\varphi_{h} \in X_{h}: \min _{x_{a} \in a} n_{a, x}\left(\psi_{h}\right) \cdot\right. & \left(\varphi_{h}\left(x_{a}\right)-\varphi_{h}(x)\right) \geq \varepsilon, \\
& \text { for all edges } a \text { and all vertices } x \text { of the mesh such that } x \notin a\},
\end{aligned}
$$

where $n_{a, x}\left(\psi_{h}\right)$ is defined for all edges $a$ and vertices $x$ of the mesh such that $x \notin a$ as the only unitary element of $\mathbb{R}^{n}$ such that

$$
\min _{x_{a} \in a} n_{a, x}\left(\psi_{h}\right) \cdot\left(\psi_{h}\left(x_{a}\right)-\psi_{h}(x)\right)=\operatorname{dist}\left(\psi_{h}(a), \psi_{h}(x)\right) \geq \varepsilon .
$$

Lemma 4.2. For all elements $\psi_{h}$ of $\mathcal{A}_{h, \varepsilon}$, the convex set $T\left(\psi_{h}\right)$ is included in $\mathcal{A}_{h, \varepsilon}$.

Proof. Let $\varphi_{h}$ be a deformation that does not belong to $\mathcal{A}_{h, \varepsilon}$. There exists $T_{1}$ and $T_{2}$ elements of $\mathcal{T}_{h}$ such that $T_{1} \cap T_{2}=\emptyset$ and $\operatorname{dist}\left(\varphi_{h}\left(T_{1}\right), \varphi_{h}\left(T_{2}\right)\right)<\varepsilon$. We introduce the deformation $\psi_{s}$ parameterized by $s \in[0,1]$ and defined by

$$
\psi_{s}=(1-s) \psi_{h}+s \varphi_{h}
$$

We also denote by $f$ the function that maps any real $s \in[0,1]$ onto the distance between $\psi_{s}\left(T_{1}\right)$ and $\psi_{s}\left(T_{2}\right)$,

$$
f(s)=\operatorname{dist}\left(\psi_{s}\left(T_{1}\right), \psi_{s}\left(T_{2}\right)\right) .
$$

The map $f$ is continuous, $f(0) \geq \varepsilon$ and $f(1)<\varepsilon$. Thus, there exists a real $s \in[0,1]$ such that $0<f(s)<\varepsilon$ (let us remark that $f(1)$ could be equal to zero, hence $s=1$ is not necessarily suitable). As the minimal distance between $\psi_{s}\left(T_{1}\right)$ and $\psi_{s}\left(T_{2}\right)$ is not zero, it is reached, up to a permutation of $T_{1}$ and $T_{2}$, for a couple of points $\left(x, x_{a}\right)$ of $T_{1} \times T_{2}$ where $x$ is a vertex of the mesh, and $x_{a}$ belongs to an edge $a$ of $\mathcal{T}_{h}$. We have

$$
n_{a, x}\left(\psi_{h}\right) \cdot\left(\psi_{s}\left(x_{a}\right)-\psi_{s}(x)\right) \leq\left|\psi_{s}\left(x_{a}\right)-\psi_{s}(x)\right|=\operatorname{dist}\left(\psi_{s}\left(T_{1}\right), \psi_{s}\left(T_{2}\right)\right)<\varepsilon .
$$

We deduce from this relation that $\psi_{s}$ does not belong to $T\left(\psi_{h}\right)$. As $\psi_{s}$ is a convex combination of $\psi_{h}$ and $\varphi_{h}$, they could not both belong to $T\left(\psi_{h}\right)$. 

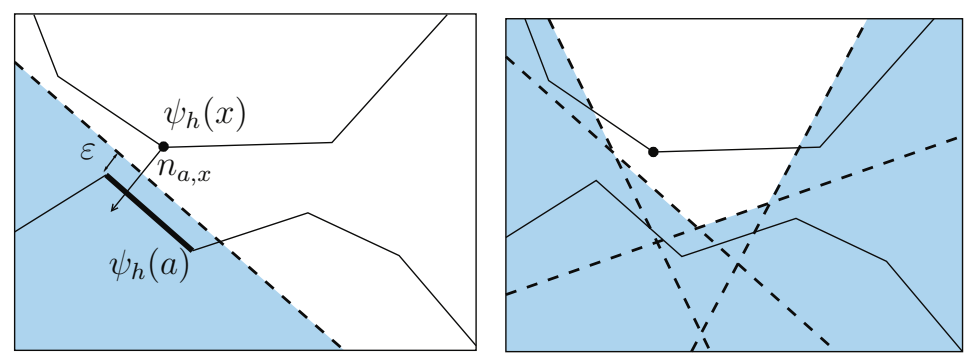

Figure 1. Constraints associated with a vertex.

Figure 1 represents the constraints imposed to a vertex $x$ of the mesh, assuming the rest of the structure remains fixed. On the left-hand side, only the constraint associated with the single edge $a$ is represented, whereas on the right-hand side all the constraints imposed by the lower structure are displayed.

\subsubsection{The three-dimensional case}

In the three-dimensional case, the definition is slightly more complex. Not only do we have to associate a constraint to each couple vertex/triangle but also to each couple edge/edge. Thus,we set

$$
\begin{aligned}
& T\left(\psi_{h}\right)=\left\{\begin{array}{l}
\varphi_{h} \in X_{h}: \min _{\substack{x_{a} \in a \\
x_{b} \in b}} n_{a, b}\left(\psi_{h}\right) \cdot\left(\varphi_{h}\left(x_{a}\right)-\varphi_{h}\left(x_{b}\right)\right) \geq \varepsilon,
\end{array}\right. \\
& \text { for all edges } a \text { and } b \text { of the mesh } \mathcal{T}_{h} \text { such that } a \cap b=\emptyset \text { and }
\end{aligned}
$$

$\min _{x_{T} \in T} n_{T, x}\left(\psi_{h}\right) \cdot\left(\varphi_{h}\left(x_{T}\right)-\varphi_{h}(x)\right) \geq \varepsilon$, for all triangles $T$ and all vertices $x$ of the mesh $\mathcal{T}_{h}$ such that $\left.x \notin T\right\}$,

where $n_{a, b}\left(\psi_{h}\right)$ and $n_{T, x}\left(\psi_{h}\right)$ are the unitary elements of $\mathbb{R}^{n}$ defined by

$$
\min _{\substack{x_{a} \in a \\ x_{b} \in b}} n_{a, b} \cdot\left(\psi_{h}\left(x_{a}\right)-\psi_{h}\left(x_{b}\right)\right)=\operatorname{dist}\left(\psi_{h}(a), \psi_{h}(b)\right)
$$

and

$$
\min _{x_{T} \in T} n_{T, x} \cdot\left(\psi_{h}\left(x_{T}\right)-\psi_{h}(x)\right)=\operatorname{dist}\left(\psi_{h}(T), \psi_{h}(x)\right) .
$$

Lemma 4.2 remains true in this case.

Remark 4.3. In the classical master/slave approach, only the triangle/vertex constraints are usually considered with the exclusion of the edge/edge type constraints. Note that mortar methods can be applied to the master/slave approach [36]. In this case, the constraints are not imposed to each single vertex, but in a weak form.

\section{Optimality COnditions}

Let us assume that the proposed algorithm converges toward an element $\varphi$ of $\mathcal{A}_{h, \varepsilon}$. Contrarily to our hopes, $\varphi$ is not, in general, a solution of the minimization problem of $J$ over $\mathcal{A}_{h, \varepsilon}$. Such a result is not surprising. Since $\mathcal{A}_{h, \varepsilon}$ is not convex, the functional $J$ may possess several local minima, even if $J$ is strongly convex. Because of the sequential nature of our algorithm, we could at most expect that a local minimum is reached. Yet this does not occur. Indeed, a fixed point of our algorithm does not necessary fulfill the optimality conditions: the action/reaction principle is partially violated. Nevertheless, the proposed algorithm is consistent in the following 
sense: all the fixed points of (3.1) verify the optimality conditions associated with the discrete minimization problem (4.1) up to a "small" error depending of the mesh size $h$ and the fixed point considered (see Prop. 5.8). Let us underline that, the problem being nonconvex, this doesn't ensure neither that fixed points of (3.1) are close to actual minimizers of the energy over the discrete admissible space $\mathcal{A}_{h, \varepsilon}$ nor that they converge toward solutions of the continuous problem as $h$ and $\varepsilon$ go to zero. For the sake of simplicity, complete proofs are only given in the two dimensional setting.

\subsection{Optimality conditions associated to the discretized problem}

\subsubsection{The two-dimensional case}

Let $\varphi$ be a solution of the minimization problem of $J$ over $\mathcal{A}_{h, \varepsilon}$. If $V$ is a small enough neighborhood of $\varphi$ in $X_{h}$, arguing as in the proof of Lemma 4.2 , it is easy to show that

$$
\mathcal{A}_{h, \varepsilon} \cap V=\left\{\varphi \in X_{h}: \min _{x_{a} \in a} \operatorname{dist}\left(\varphi\left(x_{a}\right), \varphi(x)\right) \geq \varepsilon\right.
$$

for all edges $a$ and all vertices $x$ of $\mathcal{T}_{h}$ such that $\left.x \notin a\right\} \cap V$.

For all vertices $x$ and all edges $a$ of $\mathcal{T}_{h}$, the function

$$
F_{a, x}(\psi):=\varepsilon-\operatorname{dist}(\psi(a), \psi(x))
$$

is continuously differentiable on $V$. More precisely, let us denote by $a_{0}$ and $a_{1}$ the endpoints of the edge $a$, by $p_{a, x}(\varphi)$ the element of the edge $a$ that is mapped by $\varphi$ onto the projection of $\varphi(x)$ on $\varphi(a)$ and by $\alpha_{a, x}^{0}$ and $\alpha_{a, x}^{1}$ the homogeneous barycentric coordinates of $p_{a, x}(\varphi)$ on $a$. We have

$$
\begin{gathered}
p_{a, x}(\varphi) \in a \\
p_{a, x}(\varphi)=\alpha_{a, x}^{0} a_{0}+\alpha_{a, x}^{1} a_{1} \\
\operatorname{dist}(\varphi(a), \varphi(x))=\left|\varphi\left(p_{a, x}(\varphi)\right)-\varphi(x)\right|, \\
\alpha_{a, x}^{0}+\alpha_{a, x}^{1}=1, \\
\alpha_{a, x}^{0}, \alpha_{a, x}^{1} \geq 0,
\end{gathered}
$$

and

$$
\left\langle D F_{a, x}(\varphi), \hat{\varphi}\right\rangle=n_{a, x}(\varphi) \cdot\left(\hat{\varphi}(x)-\hat{\varphi}\left(p_{a, x}(\varphi)\right)\right),
$$

where $n_{a, x}(\varphi)$ is defined by (4.3). Since $\left\langle D F_{a, x}(\varphi), \varphi\right\rangle<0$, the constraints are qualified. Hence, the optimality conditions are given by the following proposition.

Proposition 5.1. Let $\varphi: M \rightarrow \mathbb{R}^{2}$ be a solution of (4.1), that is a minimizer of $J$ over $\mathcal{A}_{h, \varepsilon}$. Assume that $J$ is differentiable, then there exists a family of nonnegative reals $\lambda_{a, x}$, where a spans the edges of $\mathcal{T}_{h}$ and $x$ its vertices, such that for any test function $\hat{\varphi} \in X_{h}$, we have

$$
\left\{\begin{array}{l}
\left\langle J^{\prime}(\varphi), \hat{\varphi}\right\rangle=\sum_{x \notin a} h \lambda_{a, x} n_{a, x} \cdot\left(\hat{\varphi}\left(p_{a, x}\right)-\hat{\varphi}(x)\right), \\
\lambda_{a, x} F_{a, x}(\varphi)=0 \\
\varphi \in \mathcal{A}_{h, \varepsilon}
\end{array}\right.
$$

where $p_{a, x}$ is defined by (5.2), $n_{a, x}$ by (4.3) and $F_{a, x}$ by (5.1).

Remark 5.2. The Lagrange multiplier $\lambda_{a, x}$ is the contact force by unit length exerted by the edge $a$ onto the point $x$ (in the reference configuration). Note that at most six edges may be in contact with a given vertex of the mesh. 
Remark 5.3. If Dirichlet conditions are imposed on part of the body $M$, the constraints may not be qualified in some - rare in practice - cases and the conclusion of Proposition 5.1 may be violated.

\subsubsection{The three-dimensional case}

In order to obtain the optimality conditions fulfilled by the solutions of (4.1) in the three-dimensional case, we can proceed exactly as in the two-dimensional case. However, the formulation is slightly more complicated, since two kinds of contacts have to be considered, not only contacts between two edges but also contacts between a vertex and a triangle. For any couple of edges $(a, b)$ of the mesh $\mathcal{T}_{h}$, we introduce the function

$$
F_{a, b}(\psi):=\varepsilon-\operatorname{dist}(\psi(a), \psi(b))
$$

Likewise, for all vertices $x$ and all triangles $T$ of the mesh $\mathcal{T}_{h}$, we set

$$
F_{T, x}(\psi):=\varepsilon-\operatorname{dist}(\psi(T), \psi(x)) .
$$

Let $\varphi$ be a solution of the minimization of $J$ over $\mathcal{A}_{h, \varepsilon}$. As in the proof of Lemma 4.2, it can be proved that if $V$ is a small enough neighborhood of $\varphi$ in $X_{h}$,

$$
\mathcal{A}_{h, \varepsilon} \cap V=\left\{\varphi \in X_{h}: F_{a, b}(\psi) \leq 0 \text { and } F_{T, x}(\psi) \leq 0\right.
$$

for all edges $a$ and $b$, all vertices $x$ and triangles $T$ of $\mathcal{T}_{h}$ such that $a \cap b=\emptyset$ and $\left.x \notin T\right\} \cap V$.

For all couples of edges $(a, b)$ of $\mathcal{T}_{h}$, we denote by $\mathcal{P}_{a, b}$ the set of couples of points belonging to $a \times b$ that minimize the distance between $\varphi(a)$ and $\varphi(b)$, that is

$$
\mathcal{P}_{a, b}:=\left\{\left(p_{a, b}, p_{b, a}\right) \in a \times b \operatorname{such} \text { that } \operatorname{dist}(\varphi(a), \varphi(b))=\left|\varphi\left(p_{a, b}\right)-\varphi\left(p_{b, a}\right)\right|\right\} .
$$

Observe that $\mathcal{P}_{a, b}$ contains a unique element, except when $\varphi(a)$ and $\varphi(b)$ are collinear. Finally, for any couple of vertex $x$ and triangle $T$, we denote by $p_{T, x}$ the element of $T$ such that

$$
\operatorname{dist}(\varphi(T), \varphi(x))=\left|\varphi\left(p_{T, x}\right)-\varphi(x)\right| \text { and } p_{T, x} \in T
$$

The function $F_{T, x}$ is differentiable and for all test functions $\hat{\varphi} \in X_{h}$, we have

$$
\left\langle D F_{T, x}(\varphi), \hat{\varphi}\right\rangle=n_{T, x}(\varphi) \cdot\left(\hat{\varphi}(x)-\hat{\varphi}\left(p_{T, x}\right)\right)
$$

On the other hand, the functions $F_{a, b}$ are not differentiable, but their (basic or limiting) subdifferentials (see [22], Def. 1.77, p. 82) are given for all $\varphi \in \mathcal{A}_{h, \varepsilon}$ by

$$
\partial F_{a, b}(\varphi)=\mathcal{C}_{a, b}(\varphi)
$$

where

$$
\mathcal{C}_{a, b}(\varphi):=\left\{L \in X_{h}^{*} \text { such that } L(\hat{\varphi})=n_{a, b}(\varphi) \cdot\left(\hat{\varphi}\left(p_{b, a}\right)-\hat{\varphi}\left(p_{a, b}\right)\right) \text { with }\left(p_{a, b}, p_{b, a}\right) \in \mathcal{P}_{a, b}\right\} .
$$

The expression (5.8) of the subdifferential of $F_{a, b}$ follows directly from the fact that the presubdifferential (or Frechet subdifferential) $\hat{\partial} F_{a, b}$ of $F_{a, b}$ is equal to the multivalued function $\mathcal{C}_{a, b}$ which is upper semicontinuous. 
Then, by the limiting representation theorem (see [22], (1.56), Thm. 1.89, p. 92), we have

$$
\begin{aligned}
\partial F_{a, b}(\varphi) & =\operatorname{Limsup}_{\delta \varphi \rightarrow 0} \hat{\partial} F_{a, b}(\varphi+\delta \varphi) \\
& =\operatorname{Limsup}_{\delta \varphi \rightarrow 0} \mathcal{C}_{a, b}(\varphi+\delta \varphi)=\mathcal{C}_{a, b}(\varphi) .
\end{aligned}
$$

We recall that the presubdifferential of $F_{a, b}$ at $\varphi$ could be defined by

$$
\hat{\partial} F_{a, b}(\varphi):=\left\{L \in X_{h}^{*} \text { such that } \liminf _{\delta \varphi \rightarrow 0} \frac{F_{a, b}(\varphi+\delta \varphi)-F_{a, b}(\varphi)-L(\delta \varphi)}{|\delta \varphi|} \geq 0\right\} .
$$

It can be easily proved to be equal to $\mathcal{C}_{a, b}(\varphi)$ using the following development

$$
F_{a, b}(\varphi+\delta \varphi)=F_{a, b}(\varphi)+\max _{L \in \mathcal{C}_{a, b}(\varphi)} L(\delta \varphi)+o(\delta \varphi) .
$$

Proving that

$$
F_{a, b}(\varphi+\delta \varphi) \geq F_{a, b}(\varphi)+\max _{L \in \mathcal{C}_{a, b}(\varphi)} L(\delta \varphi)+o(\delta \varphi)
$$

is basically trivial, whereas the converse inequality, though not difficult to derive, requires a sharper analysis. Finally, as for all admissible deformations $\varphi$, we have $\left\langle D F_{T, x}(\varphi), \varphi\right\rangle>0$ and for all non zero elements $L$ of $\partial F_{a, b}(\varphi),\langle L(\varphi), \varphi\rangle>0$, the constraints are qualified and the following optimality condition can be stated (using for instance Cor. 5.10, (5.20), p. 14 of [23]).

Proposition 5.4. Assume that $J$ is differentiable. Let $\varphi: M \rightarrow \mathbb{R}^{3}$ be a solution of the minimization problem (4.1). Then, there exist two families of non negative reals $\lambda_{a, b}$ and $\lambda_{T, x}$, where $a$ and $b$ span the set of edges of $\mathcal{T}_{h}, T$ its triangles, and $x$ its vertices, such that for any test function $\hat{\varphi} \in X_{h}$, we have

$$
\left\{\begin{array}{l}
\left\langle J^{\prime}(\varphi), \hat{\varphi}\right\rangle=\sum_{\substack{a, b \\
a \cap b=\emptyset}} h^{2} \lambda_{a, b} n_{a, b} \cdot\left(\hat{\varphi}\left(p_{a, b}-\hat{\varphi}\left(p_{b, a}\right)\right)\right)+\sum_{\substack{T, x \\
x \notin T}} h^{2} \lambda_{T, x} n_{T, x} \cdot\left(\hat{\varphi}\left(p_{T, x}\right)-\hat{\varphi}(x)\right), \\
\lambda_{T, x} F_{T, x}(\varphi)=0, \\
\lambda_{a, b} F_{a, b}(\varphi)=0, \\
\varphi \in \mathcal{A}_{h, \varepsilon},
\end{array}\right.
$$

where $\left(p_{a, b}, p_{b, a}\right) \in \mathcal{P}_{a, b}$, and $\left(p_{T, x}\right)$ is defined by (5.7).

\subsection{Optimality conditions associated with a fixed point}

In this section, we derive the optimality conditions fulfilled by a fixed point of our algorithm. Those conditions differ from the one associated with the solutions of the discrete minimization problem (4.1) obtained in the previous section. However, they remain close and every fixed point of the algorithm satisfies the optimality conditions associated with the discretized optimization problem up to a small error. For the sake of simplicity, we confine to the two-dimensional case. The three-dimensional case may be addressed in a similar way.

Proposition 5.5. Let $\varphi$ be a fixed point of the algorithm presented in Section 3 applied to the minimization of $J$ over $\mathcal{A}_{h, \varepsilon}$, where the "neighborhoods" $T(\psi)$ are defined by (4.2) or equivalently by

$$
T(\psi)=\left\{\varphi_{h} \in X_{h}: F_{a, x}^{0}\left(\varphi_{h}\right) \leq 0 \text { and } F_{a, x}^{1}\left(\varphi_{h}\right) \leq 0, \text { for any vertex } x \text { and any edge a of } \mathcal{T}_{h}\right\},
$$

where $F_{a, x}^{0}$ and $F_{a, x}^{1}$ are defined by

$$
\begin{aligned}
& F_{a, x}^{0}\left(\varphi_{h}\right)=\varepsilon-n_{a, x}(\psi) \cdot\left(\varphi_{h}\left(a_{0}\right)-\varphi_{h}(x)\right), \\
& F_{a, x}^{1}\left(\varphi_{h}\right)=\varepsilon-n_{a, x}(\psi) \cdot\left(\varphi_{h}\left(a_{1}\right)-\varphi_{h}(x)\right),
\end{aligned}
$$


and $a_{0}$ and $a_{1}$ are the endpoints of the edge $a$. Then, there exists a family $\lambda_{a, x}^{i}$ (where $i=0,1$, a is any edge of the mesh $\mathcal{T}_{h}$ and $x$ any of its vertices) of non negative reals, such that for any test function $\hat{\varphi} \in X_{h}$,

$$
\left\{\begin{array}{l}
\left\langle J^{\prime}(\varphi), \hat{\varphi}\right\rangle=\sum_{x \notin a} h n_{a, x} \cdot\left(\lambda_{a, x}^{0} \hat{\varphi}\left(a_{0}\right)+\lambda_{a, x}^{1} \hat{\varphi}\left(a_{1}\right)-\left(\lambda_{a, x}^{0}+\lambda_{a, x}^{1}\right) \hat{\varphi}(x)\right) \\
\lambda_{a, x}^{0} F_{a, x}^{0}(\varphi)=0 \\
\lambda_{a, x}^{1} F_{a, x}^{1}(\varphi)=0 \\
\varphi \in \mathcal{A}_{h, \varepsilon} .
\end{array}\right.
$$

Remark 5.6. The dependence of the functions $F_{a, x}^{0}$ and $F_{a, x}^{1}$ with respect to $\psi$ in the definition of $T(\psi)$ is implicit and does not appear in the notations used.

Proof. Let $\varphi$ be an element of $\mathcal{A}_{h, \varepsilon}$ and $\psi$ be a solution of the minimization problem

$$
\psi \in T(\varphi) ; \quad J(\psi)=\min _{\widetilde{\psi} \in T(\varphi)} J(\widetilde{\psi})
$$

As the functions $F_{a, x}^{i}$ are affine for all edges $a$, all vertices $x$ and all indexes $i \in\{0,1\}$, the constraints are always qualified. It follows that there exists a family $\left(\lambda_{a, x}^{i}\right)(i=0,1)$ of non-negative reals such that for all test functions $\hat{\varphi} \in X_{h}$, we have

$$
\left\langle J^{\prime}(\psi), \hat{\varphi}\right\rangle+\sum_{x \notin a} h n_{a, x}(\varphi) \cdot\left(\left(\lambda_{a, x}^{0}+\lambda_{a, x}^{1}\right) \hat{\varphi}(x)-\lambda_{a, x}^{0} \hat{\varphi}\left(a_{0}\right)-\lambda_{a, x}^{1} \hat{\varphi}\left(a_{1}\right)\right)=0 .
$$

Moreover,

$$
\lambda_{a, x}^{0} F_{a, x}^{0}(\psi)=\lambda_{a, x}^{1} F_{a, x}^{1}(\psi)=0
$$

for all edges $a$ and all vertices $x$ such that $x \notin a$. Thence, if $\varphi$ is a fixed point of the algorithm, it is itself a solution of (5.11) and the conclusion of Proposition 5.5 follows from (5.12)-(5.13) and the inclusion of $T(\varphi)$ into $\mathcal{A}_{h, \varepsilon}$.

Remark 5.7. Note that if the sequence $\varphi_{n}$ generated by the algorithm introduced in Section 3 converges toward an element $\varphi$, then $\varphi$ is a fixed point of the algorithm. Indeed, as $J\left(\varphi_{n}\right)$ is decreasing, we have

$$
J(\varphi) \leq J\left(\varphi_{n+1}\right)=\inf _{\psi \in T\left(\varphi_{n}\right)} J(\psi) .
$$

In follows that

$$
J(\varphi) \leq \inf _{\psi \in \cup_{n} T\left(\varphi_{n}\right)} J(\psi)
$$

and

with

$$
J(\varphi) \leq \inf _{\psi \in U(\varphi)} J(\psi)
$$

$$
U(\psi):=\left\{\varphi_{h} \in X_{h}: F_{a, x}^{0}\left(\varphi_{h}\right)<0 \text { and } \mathrm{F}_{a, x}^{1}\left(\varphi_{h}\right)<0, \text { for any vertex } x \text { and any edge } a \text { of } \mathcal{T}_{h}\right\}
$$

where $F_{a, x}^{0}$ and $F_{a, x}^{1}$ are defined with respect to $n_{a, x}(\psi)$. It is easy to check that $T(\varphi)=\overline{U(\varphi)}$, so that

$$
J(\varphi) \leq \inf _{\psi \in T(\varphi)} J(\psi)
$$

and $\varphi$ is a fixed point. 
Proposition 5.8. Every fixed point of the algorithm of the Section 3 applied to the discrete minimization problem (4.1), where the map $T(\cdot)$ is defined by (4.2) satisfies the optimality conditions (5.3) up to a small error. More precisely, if $\varphi_{h}$ is a fixed point of this algorithm, there exists a family $\lambda_{a, x}$ of non negative reals, where a spans the edges of $\mathcal{T}_{h}$, and $x$ its vertices such that, for every real $s>0$,

$$
\left\{\begin{array}{l}
\left\|R\left(\varphi_{h}, \lambda\right)\right\|_{-s, h} \leq \sqrt{C\left(\varphi_{h}\right)}\|\lambda\|_{h} h^{s / 2} \\
\lambda_{a, x} F_{a, x}\left(\varphi_{h}\right)=0 \\
\varphi_{h} \in \mathcal{A}_{h, \varepsilon}
\end{array}\right.
$$

where $R\left(\varphi_{h}, \lambda\right)$ is the residual associated to the minimization problem (4.1)

$$
\left\langle R\left(\varphi_{h}, \lambda\right), \hat{\varphi}\right\rangle=\left\langle J^{\prime}\left(\varphi_{h}\right), \hat{\varphi}\right\rangle-\sum_{x \notin a} h \lambda_{a, x} n_{a, x} \cdot\left(\hat{\varphi}\left(p_{a, x}\right)-\hat{\varphi}(x)\right)
$$

$\|\cdot\|_{h}$ is the norm associated with the Lagrange multipliers $\lambda$,

$$
\begin{gathered}
\|\lambda\|_{h}=\left(\sum_{x \notin a}\left|\lambda_{a, x}\right|^{2} h\right)^{1 / 2} \\
\|R\|_{-s, h}=\sup _{\|\hat{\varphi}\|_{s, h}=1}\|\langle R, \hat{\varphi}\rangle\|, \text { with } \quad\|\hat{\varphi}\|_{s, h}=\left(\sum_{a}\left|\frac{\hat{\varphi}\left(a_{0}\right)-\hat{\varphi}\left(a_{1}\right)}{h^{s}} h\right|^{2}\right)^{1 / 2}
\end{gathered}
$$

and $C\left(\varphi_{h}\right)$ is the constant defined by

$$
C\left(\varphi_{h}\right):=\max _{a}\left\{C_{a}\left(\varphi_{h}\right):=\operatorname{Card}\left(\left\{x \in \mathcal{T}_{h} \text { such that } F_{a, x}=0\right\}\right)\right\}
$$

In the case of a one-dimensional structure moving in $\mathbb{R}^{2}$, for $s=2$, we have

$$
\left\|R\left(\varphi_{h}, \lambda\right)\right\|_{-s, h}=\left\|R\left(\varphi_{h}, \lambda\right)\right\|_{H^{-1}\left(M ; \mathbb{R}^{2}\right)},
$$

and Proposition 5.8 leads to the estimate

$$
\left\|R\left(\varphi_{h}, \lambda\right)\right\|_{H^{-1}\left(M ; \mathbb{R}^{2}\right)} \leq \sqrt{C\left(\varphi_{h}\right)}\|\lambda\|_{h} h
$$

and the error made on the residual due to the contact algorithm is of the same order as the error due to the $P_{1}$ finite element discretization. For a two dimensional structure $M$ moving in $\mathbb{R}^{2}$, it is usually enough to handle contacts on its boundary $\partial M$. In this case, Proposition 5.8 with $s=1 / 2$ leads to

$$
\left|\left\langle R\left(\varphi_{h}, \lambda\right), \hat{\varphi}\right\rangle\right| \leq \sqrt{C\left(\varphi_{h}\right)}\|\lambda\|_{h} h^{1 / 2}\|\hat{\varphi}\|_{H^{1 / 2}\left(\partial M ; \mathbb{R}^{2}\right)}
$$


From the theorem of trace, there exists a constant $C^{\prime}$ such that $\|\hat{\varphi}\|_{H^{1 / 2}\left(M ; \mathbb{R}^{2}\right)} \leq C^{\prime}\|\hat{\varphi}\|_{H^{1}\left(M ; \mathbb{R}^{2}\right)}$, and we obtain that

that is

$$
\left|\left\langle R\left(\varphi_{h}, \lambda\right), \hat{\varphi}\right\rangle\right| \leq C^{\prime} \sqrt{C\left(\varphi_{h}\right)}\|\lambda\|_{h} h^{1 / 2}\|\hat{\varphi}\|_{H^{1}\left(M ; \mathbb{R}^{2}\right)},
$$

$$
\left\|R\left(\varphi_{h}, \lambda\right)\right\|_{H^{-1}\left(M ; \mathbb{R}^{2}\right)} \leq C^{\prime} \sqrt{C\left(\varphi_{h}\right)}\|\lambda\|_{h} h^{1 / 2} .
$$

Remark 5.9. We recall that the Lagrange multipliers $\lambda_{a, x}$ represent the linear force exerted by a part of the solid on another. The sum which defines the norm $\|\lambda\|_{h}$ contains $C h^{-2}$ elements so that, at the first glance, this sum may not remain bounded as the discretization gets finer. Fortunately, the number of edges in contact with one vertex is bounded independently of $h$. Thus, the sum which defined $\|\lambda\|_{h}$ only contains a number of nonzero elements of order at most $h^{-1}$. This justifies the normalization by $h\left(\right.$ and not $h^{2}$ ) used in the definition of the norm $\|\cdot\|_{h}$.

Remark 5.10. Assume that the sequence $\varphi_{h}$ converges toward a regular deformation $\varphi$ (as $h$ and $\varepsilon$ go to zero) and that a uniform mesh is used, then the number of vertices $C_{a}\left(\varphi_{h}\right)$ in contact with one edge $a$ is of the order

$$
C_{a}\left(\varphi_{h}\right) \simeq \sum_{\substack{x \in \varphi^{-1}(\varphi(y)) \\ x \neq y}}|\dot{\varphi}(y)| /|\dot{\varphi}(x)|,
$$

where $y$ is an element of the edge $a$. In such a situation, $C\left(\varphi_{h}\right)$ remains bounded.

Proof. The deformation $\varphi_{h}$ verifies the optimality conditions given by Proposition 5.5. We set $\lambda_{a, x}=\lambda_{a, x}^{0}+\lambda_{a, x}^{1}$, then

$$
\begin{aligned}
\left\langle R\left(\varphi_{h}, \lambda\right), \hat{\varphi}\right\rangle & =\sum_{x \notin a} h n_{a, x} \cdot\left(\left(\lambda_{a, x}^{0}-\alpha_{a, x}^{0} \lambda_{a, x}\right) \hat{\varphi}\left(a_{0}\right)+\left(\lambda_{a, x}^{1}-\alpha_{a, x}^{1} \lambda_{a, x}\right) \hat{\varphi}\left(a_{1}\right)\right) \\
& =\sum_{x \notin a} h n_{a, x} \cdot\left(\hat{\varphi}\left(a_{0}\right)-\hat{\varphi}\left(a_{1}\right)\right)\left(\alpha_{a, x}^{1} \lambda_{a, x}^{0}-\alpha_{a, x}^{0} \lambda_{a, x}^{1}\right) \\
& \leq \sum_{a}\left(\sum_{x \notin a} h\left|\alpha_{a, x}^{1} \lambda_{a, x}^{0}-\alpha_{a, x}^{0} \lambda_{a, x}^{1}\right|^{2}\right)^{1 / 2}\left(\sum_{\substack{x \notin a \\
\lambda_{a, x} \neq 0}} h\left|\hat{\varphi}\left(a_{0}\right)-\hat{\varphi}\left(a_{1}\right)\right|^{2}\right)^{1 / 2} \\
& \leq \sum_{a}\left(\sum_{x \notin a} h\left|\lambda_{a, x}\right|^{2}\right)^{1 / 2}\left(C_{a}\left(\varphi_{h}\right) h\left|\hat{\varphi}\left(a_{0}\right)-\hat{\varphi}\left(a_{1}\right)\right|^{2}\right)^{1 / 2} \\
& \leq\|\lambda\|_{h} \sqrt{C\left(\varphi_{h}\right)}\left(\sum_{a} \frac{\left|\hat{\varphi}\left(a_{0}\right)-\hat{\varphi}\left(a_{1}\right)\right|^{2}}{h^{s}} h\right)^{1 / 2} h^{s / 2} .
\end{aligned}
$$

Moreover, since $\varphi_{h}$ is a fixed point, we have

$$
F_{a, x}\left(\varphi_{h}\right)=\min \left(F_{a, x}^{0}\left(\varphi_{h}\right), F_{a, x}^{1}\left(\varphi_{h}\right)\right) .
$$

If $\lambda_{a, x} \neq 0$, we either have $\lambda_{a, x}^{0} \neq 0$ or $\lambda_{a, x}^{1} \neq 0$. Then, from the optimality conditions satisfied by $\varphi_{h}$, we infer that either $F_{a, x}^{0}(\varphi)$ or $F_{a, x}^{1}(\varphi)$ is equal to zero. From (5.14), it follows that $F_{a, x}\left(\varphi_{h}\right)=0$, and that for any couple vertex/edge $(x, a)$, we have

$$
F_{a, x}\left(\varphi_{h}\right) \lambda_{a, x}=0
$$


Remark 5.11. In the $1 \mathrm{~d}-2 \mathrm{~d}$ case, the error made on the residual due to the contact algorithm is of the same order as the error due to the $P_{1}$ finite element discretization. Though it is possible to use an alternate definition of the "neighborhood" $T(\cdot)$ so that all fixed points of our algorithm exactly match the optimality conditions associated to the discrete minimization problem of $J$ on $\mathcal{A}_{h, \varepsilon}$, it will not increase the precision of the global scheme.

\section{The Dynamic CASE}

We can extend our analysis to the study of dynamic systems with frictionless contacts and soft impacts. Our method can be applied to most time-discretization schemes in order to take into account the contact constraint. Let us give a simple example. In the dynamic case, the evolution of a family of deformable bodies can be approximated by a sequence of minimization problems defined on the set of admissible deformations. For instance, the implicit finite difference method using a backward difference for the time discretization consists in computing at each time step $t_{i+1}=(i+1)(\Delta t)$ the solution of the minimization problem

$$
\min _{\varphi_{i+1} \in \mathcal{A}_{h, \varepsilon}} \frac{1}{2} \int_{M} \rho\left(v_{i+1}-v_{i}\right)^{2} \mathrm{~d} x+J\left(\varphi_{i+1}\right),
$$

where $v_{i+1}=\left(\varphi_{i+1}-\varphi_{i}\right) / \Delta t$, and $\rho$ is the inertial mass per volume unit. Each of those problems can be solved using our algorithm. Naturally, other time discretization schemes can be used (like Newmark for instance).

\section{Numerical EXAMPLES}

Our algorithm has been applied to handle contacts between aortic valves in a three dimensional setting [1]. In this section, we present different applications of our method in the more simple two-dimensional case. We give four different examples. In the first one, we compute the equilibrium state of inflated balloons (1d-2d static case). In the second example, we consider such balloons placed in a rotating box ( $1 \mathrm{~d}-2 \mathrm{~d}$ dynamic case). A $2 \mathrm{~d}-2 \mathrm{~d}$ static case is treated in the third example (linear elastic $2 \mathrm{~d}$ bodies). The last example is dedicated to a case with self-contacts (equilibrium state of a nonlinear elastic beam under compression).

\subsection{1d-2d static case}

We consider a system made of elastic membranes $M_{i}$, diffeomorphic to the interval $[0,1]$ that contains a gas. The stored elastic energy of a membrane $M_{i}$ is defined by

$$
W_{i}(F)=\mu_{i} \begin{cases}\left(|F|^{2}-1\right)^{2} & \text { if }|F| \geq 1 \\ 0 & \text { if }|F|<1\end{cases}
$$

where $\mu_{i}>0$ is an elasticity coefficient. The internal energy of a deformation $\psi_{i}$ of the membrane $M_{i}$ is

$$
E_{i}\left(\psi_{i}\right)=\int_{M_{i}} W_{i}\left(\dot{\psi}_{i}\right) \mathrm{d} x
$$

Moreover, we assume that each membrane is fixed on a plane support and that the space between the membranes and the supports is filled with a perfect gas, which exerts on each membrane a uniform pressure inversely proportional to the area $V_{i}$ it is occupying. Thus, the total energy associated to the deformations $\left(\psi_{i}\right)=\psi$ of the membranes is

$$
I(\psi)=\sum_{i} \int_{M_{i}} W_{i}\left(\dot{\psi}_{i}\right) \mathrm{d} x-C_{i} \ln \left(V_{i}\right),
$$

where $C_{i}$ are positive constants depending on the quantity of gas contained in each membrane $M_{i}$. Any equilibrium position of the membranes is a critical points of the energy over the set of admissible deformations. 

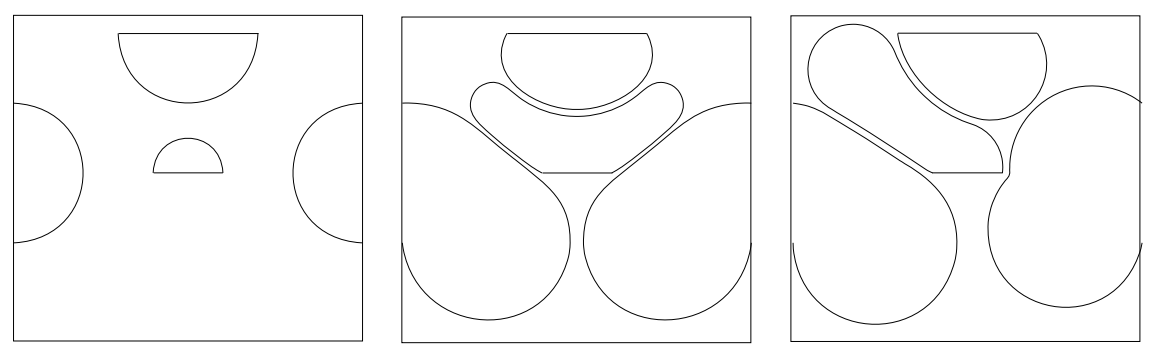

FigurE 2. Different steps of the minimization of the energy of a system made of inflated elastic balloons.

As the functions $W_{i}$ are convex, there exists a configuration $\varphi$ that minimizes the energy over the set of admissible deformations,

We solve the discretized version of this problem

$$
I(\varphi)=\inf _{\psi \in \mathcal{A}(M)} I(\psi) .
$$

$$
I\left(\varphi_{h}\right)=\inf _{\psi_{h} \in \mathcal{A}_{h, \varepsilon}(M)} I\left(\psi_{h}\right),
$$

using our method. Let us recall the steps of our algorithm:

(1) Initialization of $\varphi_{0}$ by an admissible deformation.

(2) For all $n \geq 0$, we denote by $\varphi_{n+1} \in T\left(\varphi_{n}\right)$ the solution of the minimization problem

$$
I\left(\varphi_{n+1}\right):=\inf _{\psi \in T\left(\varphi_{n}\right)} I(\psi)
$$

where $T(\cdot)$ maps any admissible deformation $\psi_{h}$ onto the convex subset of the admissible set $\mathcal{A}_{h, \varepsilon}(M)$ defined by

$$
\begin{array}{r}
T\left(\psi_{h}\right)=\left\{\varphi_{h} \in X_{h}: n_{a, x}\left(\psi_{h}\right) \cdot\left(\varphi_{h}(a)-\varphi_{h}(x)\right) \geq \varepsilon\right. \\
\text { for all edges } a \text { and all vertices } x \text { of the mesh such that } x \notin a\},
\end{array}
$$

where $n_{a, x}\left(\psi_{h}\right)$ is defined for each edge $a$ and each vertex $x$ of the mesh such that $x \notin a$ by

$$
\min _{x_{a} \in a} n_{a, x}\left(\psi_{h}\right) \cdot\left(\psi_{h}\left(x_{a}\right)-\psi_{h}(x)\right)=\operatorname{dist}\left(\psi_{h}(a), \psi_{h}(x)\right) \geq \varepsilon
$$

(3) STOP when $I\left(\varphi_{n+1}\right) \simeq I\left(\varphi_{n}\right)$.

We solve each minimization problem (7.2) with the classical Uzawa's algorithm. Figure 2 shows different steps of the minimization process. Here, four membranes are considered. After few iterations of the algorithm, an unstable symmetric equilibrium state is reached (drawn on the center of Fig. 2), then the symmetry is broken leading to a stable equilibrium state (right part of the figure).

\subsection{1d-2d dynamic case}

As mentioned in Section 6, our contact algorithm can be applied to the dynamic case, using for instance the scheme (6.1) for time discretization. Figure 3 displays for several time steps the position of elastic inflated balloons placed in a rotating box and submitted to their own weight. In this simulation, the parameter $\varepsilon$ has been chosen very small with respect to the size of the balloons (so that the gap between the balloons is not perceivable). The rotation speed of the box is constant in time. The balloons are dropped with no initial 

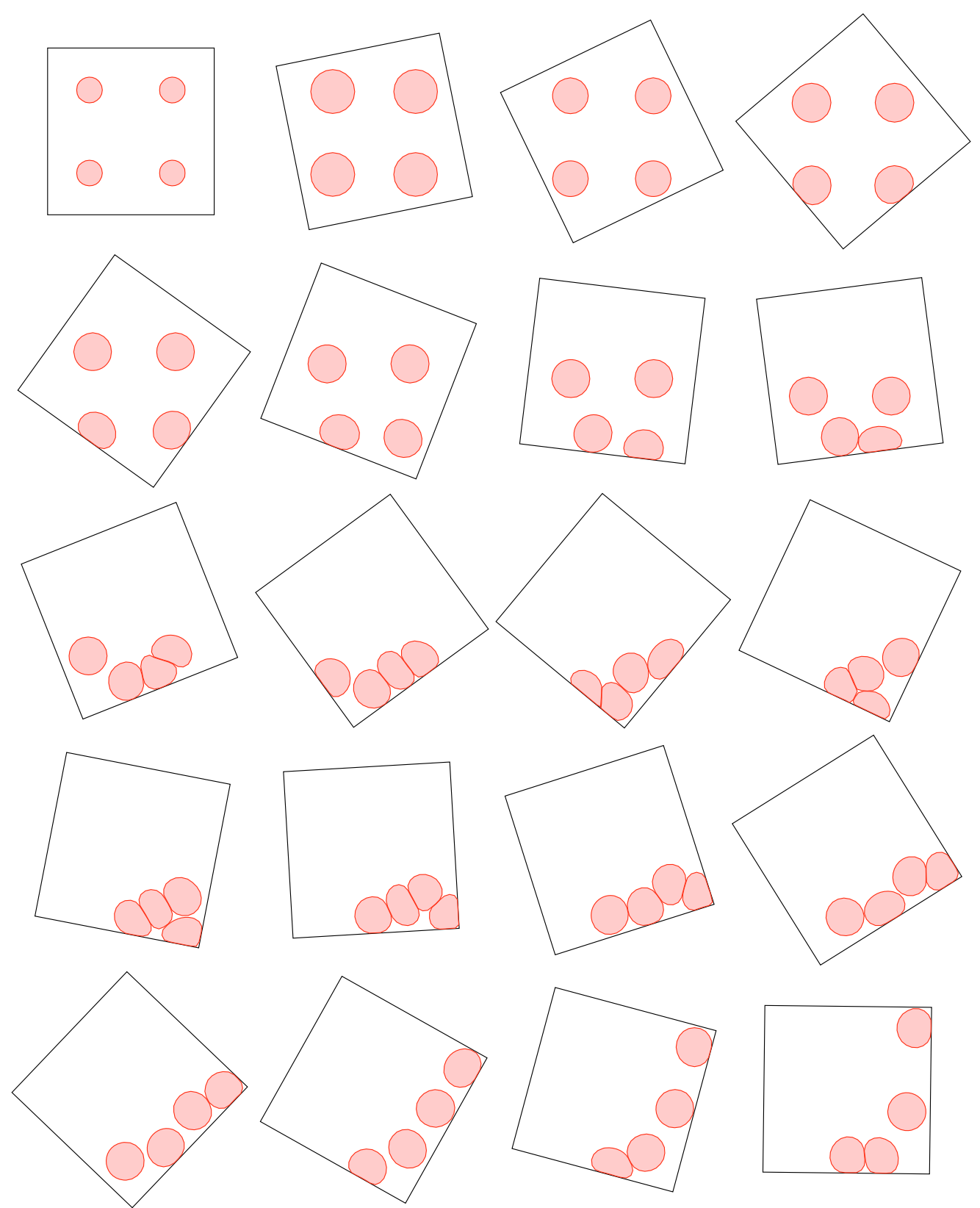

FiguRE 3. Time evolution of inflated elastic balloons submitted to their weight in a rotating box.

velocity with their membrane in the natural state. During the first time steps, the balloons, while falling, swells because of the non zero internal pressure. Ones they reach the walls of the box, they bump one into the other.

\section{3. $2 \mathrm{~d}-2 \mathrm{~d}$ static case}

In this simulation, we consider the case of two linear elastic bodies $M_{1}$ and $M_{2}$, which reference configuration are the squares

$$
\left.M_{1}=\right] 0,1[\times]-1,0\left[, \quad M_{2}=\right] 0,1[\times] 0,1[.
$$



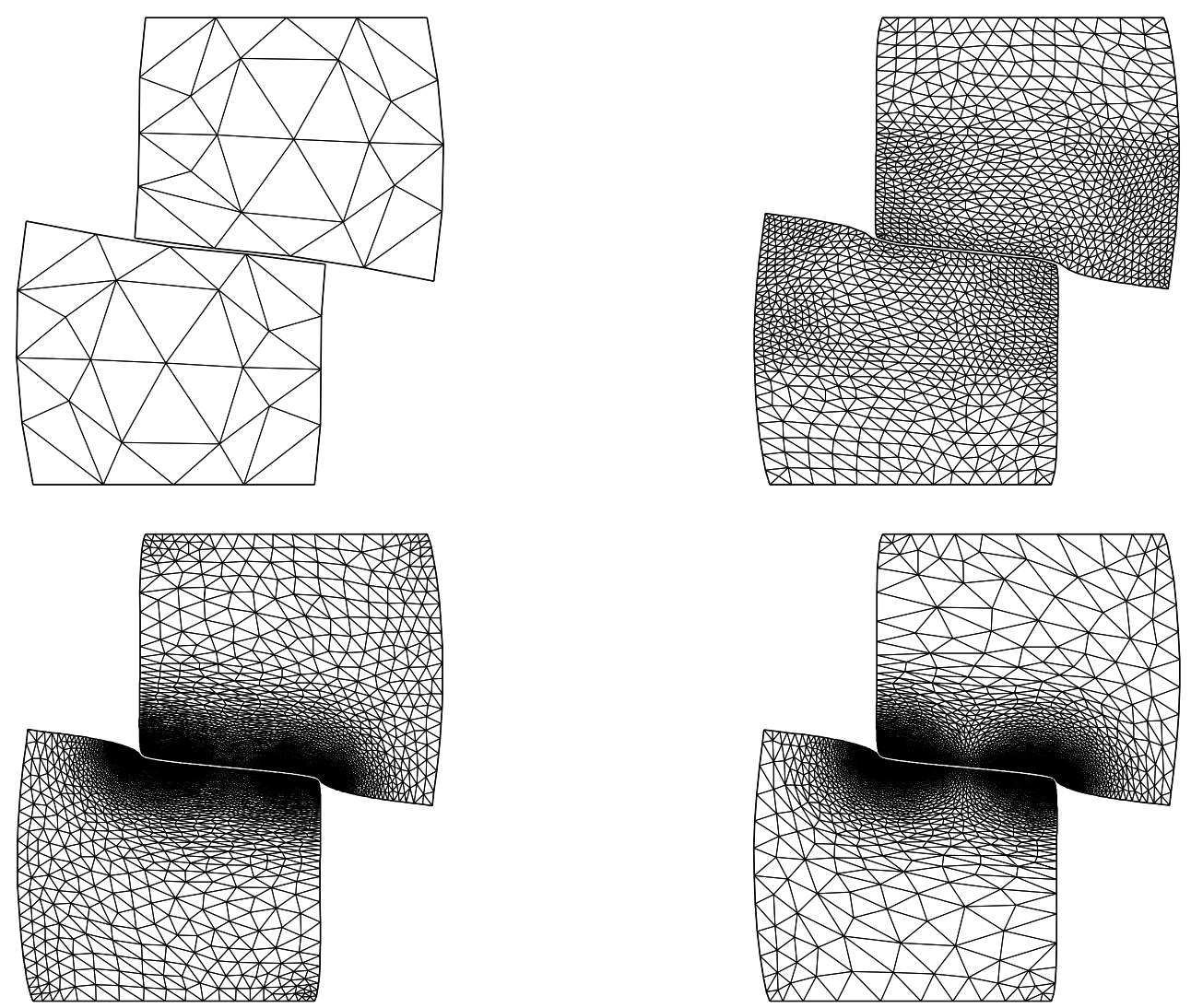

FiguRE 4. Equilibrium state for different adapted meshes of linear elastic squares squeezed one against the other.

The Young Moduli $E$ as been chosen equal to 1 , the Poisson ratio $\nu$ to 0.35 and the minimal gap distance $\varepsilon$ to $10^{-2}$. No external loads are applied, but Dirichlet conditions are imposed to the lower and upper sides of the squares $M_{1}$ and $M_{2}$ respectively

$$
\varphi(x)=(0.4,-0.4) \text { on }] 0,1[\times\{1\}
$$

and

$$
\varphi(x)=0 \text { on }] 0,1[\times\{-1\} .
$$

Our algorithm has been coupled with a mesh adaptation procedure in order to refine the computation around the contact area. Figure 4 displays the computed equilibrium states for different adapted meshes (the mesh on the left top is the most coarse, the mesh on the bottom right is the finer around the contact area). A zoom has been performed around the contact area (see Fig. 5). It enables to visualize the scale of the size of mesh and of the gap between the two bodies. One can check that the contact forces are normal to the contact surface and of same intensity on either side (see Fig. 6).

\section{4. $2 \mathrm{~d}-2 \mathrm{~d}$ case with self-contacts}

In this last example, we compute the equilibrium state of a nonlinear elastic beam under compression. The reference configuration of the beam is the open set $\Omega=] 0, L_{x}[\times] 0, L_{y}\left[\right.$, with $L_{x}=0.1, L_{y}=5$. It is made 


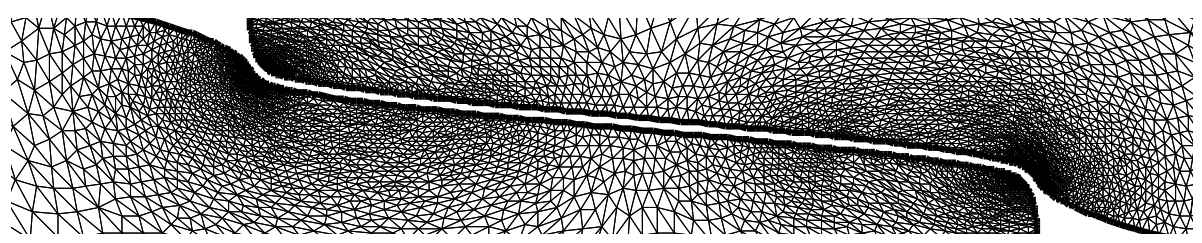

Figure 5. Contact area between the two elastic bodies.
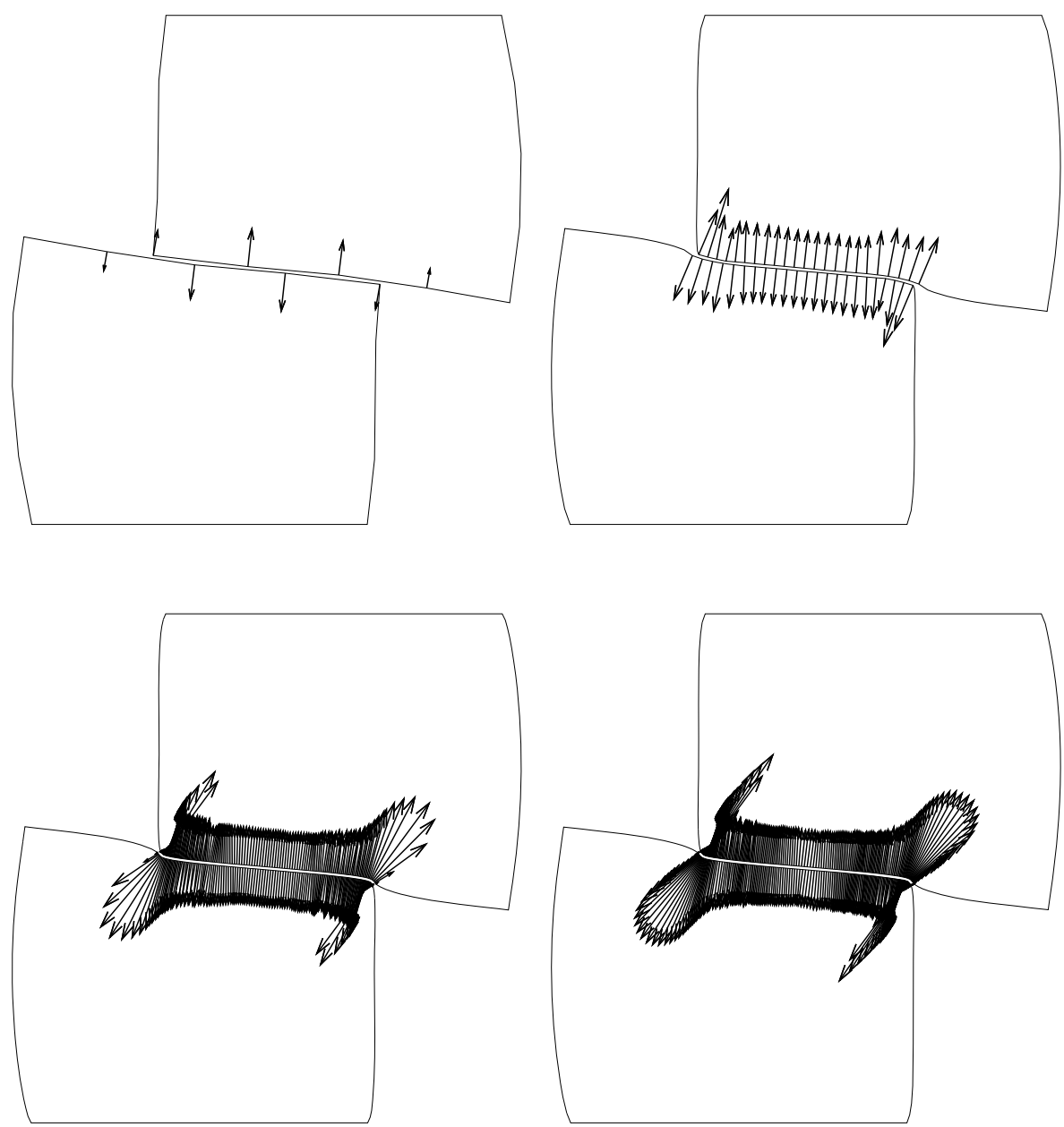

Figure 6. Computed contact forces between to linear elastic squares squeezed one against the other for different adapted meshes (see Fig. 4 for the corresponding meshes).

of a nonlinear elastic material of Saint Venant-Kirchhoff. The Young Moduli $E$ as been chosen equal to 1, the Poisson ratio $\nu$ to 0.35 . We recall that the elastic energy of a deformation $\varphi$ is given by

$$
J(\varphi)=\int_{\Omega} W_{N L}(\nabla \varphi) \mathrm{d} x,
$$



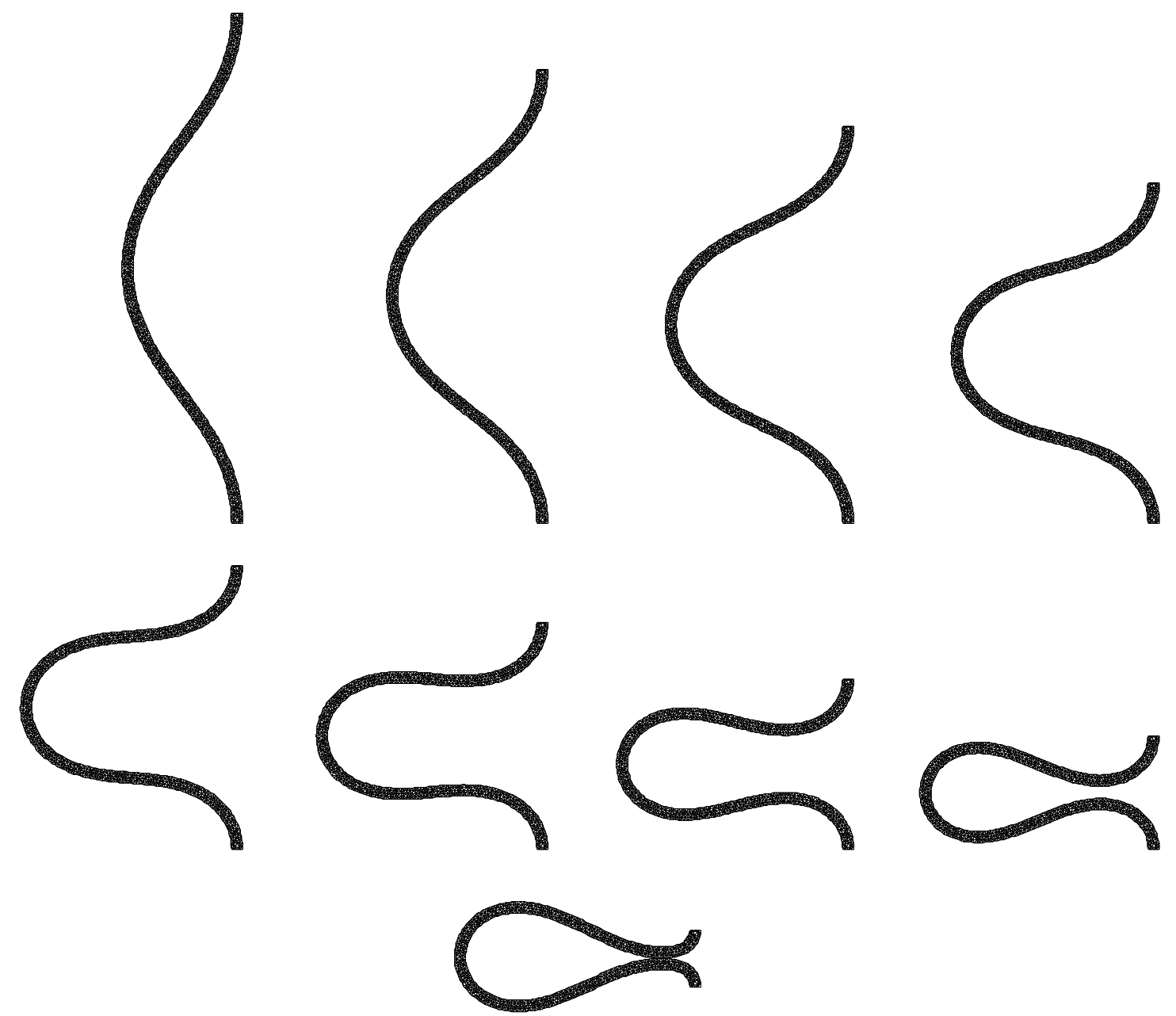

FiguRE 7. Equilibrium states of a nonlinear elastic beams compressed along the $y$-axis.

where

$$
W_{N L}(F)=\frac{\mu}{4} \operatorname{Tr}\left(\left(F^{T} F-\mathrm{Id}\right)^{2}\right)+\frac{\lambda}{8}\left(\operatorname{Tr}\left(F^{T} F-\mathrm{Id}\right)\right)^{2},
$$

with

$$
\lambda=\frac{E \nu}{(1+\nu)(1-2 \nu)} \quad \text { and } \quad \mu=\frac{E}{2(1+\nu)} .
$$

We apply the following Dirichlet conditions to the top and bottom of the beam

$$
\begin{cases}\varphi(x)=x & \text { on }] 0, L_{x}[\times\{0\} \\ \varphi(x)=\left(x_{1}, \gamma x_{2}\right) & \text { on }] 0, L_{x}\left[\times\left\{L_{y}\right\}\right.\end{cases}
$$

where $\gamma$ is a real belonging to ]0,1[. The different equilibrium states obtained are displayed in Figure 7, with $\gamma \in\{0.9,0.8,0.7,0.6,0.5,0.4,0.3,0.2,0.1\}$ ( $\gamma$ is decreasing from left to right and top to bottom). Self-contacts appear only in the last case for $\gamma=0.1$. We compare the solution obtained with the case where self-contacts are not taken into account (see Fig. 8). 

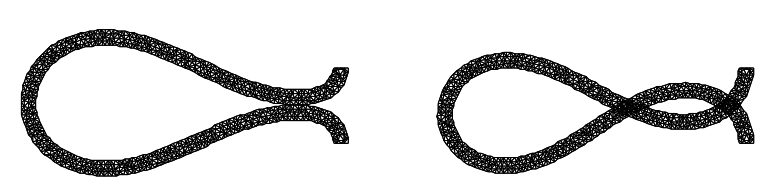

FIGURE 8. Comparison of the equilibrium states obtained with $\gamma=0.1$ when self-contacts are taken into account (left) or not (right).

\section{Conclusion}

The main advantage of the method proposed in this article in order to take into account frictionless contacts or self-contacts between deformable bodies is its robustness. During the numerical simulations we have performed, never was the classical chatter problem, that undermines many other algorithms, encountered. The drawback of our method lies in the resolution of a minimization problem with $N^{2}$ constraints (where $N$ is the number of elements of our discretization) at each step, which is prohibitive as the number of elements of the mesh becomes important. Moreover, the number of steps also depends on the size of the discretization, and is of order $\max (h, \varepsilon)^{-1}$. In the case of self-contacts, $\varepsilon$ has to be chosen smaller than $h$, which entails that we have to solve about $N$ minimization problems with $N^{2}$ constraints each. Nevertheless, it is possible to drastically reduce the computational time by regrouping the elements in bundles, and imposing non-intersection constraints to their convex hull. Moreover, in the present form, our algorithm does not take into account the rigid case. In such a case, the natural variables are the position of the gravity center, and the orientation of the solid (and not, for polygonal shapes, the position of the vertices). Thus, the definition of the "neighborhoods" $T(\cdot)$ can not be trivially extended since they are not convex sets with respect to those variables. The rigidity constraints introduce a new nonlinearity, which has to be treated on its own. Finally, in order to consider realistic applications, mechanical phenomena involved during the contact have also to be taken into account, in particular friction. It seems that classical treatments of friction should be easily adapted to our approach.

Acknowledgements. I am very grateful to Karim Trabelsi for his careful reading of the manuscript and the different improvements he suggested.

\section{REFERENCES}

[1] M. Astorino, J.-F. Gerbeau, O. Pantz and K.-F. Traoré, Fluid-structure interaction and multi-body contact: Application to aortic valves. Comput. Methods Appl. Mech. Eng. 198 (2009) 3603-3612.

[2] J.M. Ball, Global invertibility of Sobolev functions and the interpenetration of matter. Proc. Roy. Soc. Edinburgh Sect. A $\mathbf{8 8}$ (1981) 315-328.

[3] D. Baraff, Analytical methods for dynamic simulation of non-penetrating rigid bodies, in SIGGRAPH '89: Proceedings of the 16th annual conference on computer graphics and interactive techniques, ACM Press, New York, USA (1989) $223-232$.

[4] D. Baraff, Fast contact force computation for nonpenetrating rigid bodies, in SIGGRAPH '94: Proceedings of the 21st annual conference on computer graphics and interactive techniques, ACM Press, New York, USA (1994) 23-34.

[5] D. Baraff and A. Witkin, Dynamic simulation of non-penetrating flexible bodies, in SIGGRAPH '92: Proceedings of the 19th annual conference on computer graphics and interactive techniques, ACM Press, New York (1992) 303-308.

[6] D. Baraff and A. Witkin, Large steps in cloth simulation, in SIGGRAPH '98: Proceedings of the 25th annual conference on computer graphics and interactive techniques, ACM Press, New York (1998) 43-54.

[7] P.G. Ciarlet and J. Nečas, Injectivity and self-contact in nonlinear elasticity. Arch. Rational Mech. Anal. 97 (1987) $171-188$.

[8] M. Giaquinta, G. Modica and J. Souček, Cartesian currents, weak diffeomorphisms and existence theorems in nonlinear elasticity. Arch. Rational Mech. Anal. 106 (1989) 97-159.

[9] M. Giaquinta, G. Modica and J. Souček, Erratum and addendum to: "Cartesian currents, weak diffeomorphisms and existence theorems in nonlinear elasticity" [Arch. Rational Mech. Anal. 106 (1989) 97-159; MR 90c:58044]. Arch. Rational Mech. Anal. 109 (1990) 385-392.

[10] O. Gonzalez, J.H. Maddocks, F. Schuricht and H. von der Mosel, Global curvature and self-contact of nonlinearly elastic curves and rods. Calc. Var. Partial Differ. Equ. 14 (2002) 29-68. 
[11] J.O. Hallquist, G.L. Goudreau and D.J. Benson, Sliding interfaces with contact-impact in large-scale Lagrangian computations. Comput. Methods Appl. Mech. Eng. 51 (1985) 107-137.

[12] M. Heinstein, S. Attaway, J. Swegle and F. Mello, A general-purpose contact detection algorithm for nonlinear structural analysis code. Sandia Report SAND92-2141, Sandia National Laboratories, Alburquerque (1993).

[13] G. Hirota, S. Fisher and A. State, An improved finite-element contact model for anatomical simulations. Vis. Comput. 19 (2003) 291-309.

[14] M. Jean, The non-smooth contact dynamics method. Computational modeling of contact and friction. Comput. Methods Appl. Mech. Eng. 177 (1999) 235-257.

[15] M. Jean, V. Acary and Y. Monerie, Non-smooth contact dynamics approach of cohesive materials. Non-smooth mechanics. R. Soc. Lond. Philos. Trans. Ser. A Math. Phys. Eng. Sci. 359 (2001) 2497-2518.

[16] N. Kikuchi and J.T. Oden, Contact problems in elasticity: a study of variational inequalities and finite element methods, SIAM Studies in Applied Mathematics 8. Society for Industrial and Applied Mathematics (SIAM), Philadelphia (1988).

[17] A. Klarbring, Large displacement frictional contact: a continuum framework for finite element discretization. Eur. J. Mech. A Solids 14 (1995) 237-253.

[18] T.A. Laursen, Formulation and treatment of frictional contact problems using finite elements. SUDAM Report 92 (1992).

[19] T.A. Laursen, Computational contact and impact mechanics, Fundamentals of modeling interfacial phenomena in nonlinear finite element analysis. Springer-Verlag, Berlin (2002).

[20] T.A. Laursen and J.C. Simo, A continuum-based finite element formulation for the implicit solution of multibody, large deformation frictional contact problems. Int. J. Numer. Methods Eng. 36 (1993) 3451-3485.

[21] V.J. Milenkovic and H. Schmidl, Optimization-based animation, in SIGGRAPH '01: Proceedings of the 28th annual conference on computer graphics and interactive techniques, ACM Press, New York (2001) 37-46.

[22] B.S. Mordukhovich, Variational analysis and generalized differentiation. I Basic theory, Grundlehren der Mathematischen Wissenschaften 330. Springer-Verlag, Berlin (2006).

[23] B.S. Mordukhovich, Variational analysis and generalized differentiation. II Applications, Grundlehren der Mathematischen Wissenschaften 331. Springer-Verlag, Berlin (2006).

[24] J.J. Moreau, An introduction to unilateral dynamics. Lect. Notes Appl. Comput. Mech. 14 (2004) 1-26.

[25] O. Pantz, The modeling of deformable bodies with frictionless (self-)contacts. Rapport Interne 585, CMAP, École Polytechnique, Palaiseau (2005).

[26] O. Pantz, Contacts en dimension 2: Une méthode de pénalisation. Rapport Interne 597, CMAP, École Polytechnique, Palaiseau (2006).

[27] O. Pantz, The modeling of deformable bodies with frictionless (self-)contacts. Arch. Rational Mech. Anal. 188 (2008) $183-212$.

[28] M.A. Puso and T.A. Laursen, A 3D contact smoothing method using Gregory patches. Int. J. Numer. Methods Eng. 54 (2002) 1161-1194.

[29] F. Schuricht, A variational approach to obstacle problems for shearable nonlinearly elastic rods. Arch. Rational Mech. Anal. 140 (1997) $103-159$.

[30] F. Schuricht, Regularity for shearable nonlinearly elastic rods in obstacle problems. Arch. Rational Mech. Anal. 145 (1998) 23-49.

[31] F. Schuricht, Variational approach to contact problems in nonlinear elasticity. Calc. Var. Partial Differ. Equ. 15 (2002) 433-449.

[32] F. Schuricht and H. von der Mosel, Euler-Lagrange equations for nonlinearly elastic rods with self-contact. Arch. Rational Mech. Anal. 168 (2003) 35-82.

[33] Q. Tang, Almost-everywhere injectivity in nonlinear elasticity. Proc. Roy. Soc. Edinburgh Sect. A 109 (1988) 79-95.

[34] P. Wriggers, Finite element algorithms for contact problems. Arch. Comput. Methods Eng. 2 (1995) 1-49.

[35] P. Wriggers, Computational Contact Mechanics. Springer, New York (2006).

[36] B. Yang, T.A. Laursen and X. Meng, Two dimensional mortar contact methods for large deformation frictional sliding. Int. J. Numer. Methods Eng. 62 (2005) 1183-1225. 